\title{
A pragmatic approach for human risk assessment of chemical mixtures
}

Boberg, Julie; Dybdahl, Marianne; Petersen, Annette; Hass, Ulla; Svingen, Terje; Vinggaard, Anne Marie

\section{Published in:}

Current Opinion in Toxicology

Link to article, DOI:

10.1016/j.cotox.2018.11.004

Publication date:

2019

Document Version

Peer reviewed version

Link back to DTU Orbit

Citation (APA):

Boberg, J., Dybdahl, M., Petersen, A., Hass, U., Svingen, T., \& Vinggaard, A. M. (2019). A pragmatic approach for human risk assessment of chemical mixtures. Current Opinion in Toxicology, 15, 1-7.

https://doi.org/10.1016/j.cotox.2018.11.004

\section{General rights}

Copyright and moral rights for the publications made accessible in the public portal are retained by the authors and/or other copyright owners and it is a condition of accessing publications that users recognise and abide by the legal requirements associated with these rights.

- Users may download and print one copy of any publication from the public portal for the purpose of private study or research.

- You may not further distribute the material or use it for any profit-making activity or commercial gain

- You may freely distribute the URL identifying the publication in the public portal 


\section{A pragmatic approach for human risk assessment of chemical mixtures}

2 Julie Boberg, Marianne Dybdahl, Annette Petersen, Ulla Hass, Terje Svingen, Anne Marie Vinggaard

3

4

5

7

8

Corresponding author: Anne Marie Vinggaard, annv@food.dtu.dk

Keywords: Risk assessment, mixtures, toxicology, hazard index. 


\section{Abstract}

Humans are continuously exposed to complex chemical mixtures from foods and the environment. Due to our inadequate understanding of mixture effects, tools to assess the combined risk of mixed chemical exposures have been difficult to develop. In recent years, regulatory authorities across the world have made considerable progress towards developing pragmatic frameworks to deal with combined exposure to multiple chemicals for risk assessment purposes. These approaches require a high level of information about chemical exposures and toxicities, information that often is lacking. We see this data gap as delaying urgently needed improvements in chemical safety. Herein, we present a pragmatic step-by-step procedure for mixture risk assessment and propose tools for grouping of chemicals. Until we have a better understanding of adverse outcome pathways, we suggest that grouping of chemicals for mixture risk assessment be based on integrated in vivo and in vitro data, read-across as well as computational methods such as QSAR models or integrative systems biology. These latter methods can be used to predict inherent hazards or modes/mechanisms of action and to group the chemicals in cases, where no experimental data exist.

\section{Introduction}

Current regulatory approaches for assessing chemicals typically evaluate one chemical at a time, which fails to take into account the human real-world scenario of low-dose exposures to multiple chemicals. Since a growing body of evidence now suggests that simultaneous exposure to many chemicals at doses not singularly causing any effects can add up to induce adverse outcomes [1,2], the current regulatory approaches are inadequate. Due to the complexity of the issue, the implementation of methodologies for risk assessment of chemical cocktails remains a challenge, hindering the urgent need to improve chemical risk assessments. Here, we present a pragmatic step-by-step procedure for mixture risk assessment (MRA) and propose approaches for grouping of chemicals. 


\section{Current status of mixture risk assessment}

\subsection{Additive mixture effects}

Experiments have shown that chemicals, when exerting similar effects, typically act in a dose-additive manner, and far less frequently in synergistic or antagonistic ways [3,4]. This means that the efficacies of chemicals in a mixture can be added together, as long as the potency of the individual chemicals is appropriately accounted for. As dose-response curves are usually sigmoidal, the combination of many chemicals at low levels (located at the lowest horizontal part of the dose-response curve) can give rise to a pronounced adverse mixture effect (corresponding to a move up to the steeper part of the dose-response curve). This phenomenon has been observed in cellular systems [5] and animal studies [6-8].

Synergistic or antagonistic interactions are well-known occurrences in drug therapy, where it is common to see drugs either enhancing or inhibiting each other's effect, primarily due to toxicokinetic mechanisms [9]. In contrast, kinetic interactions are much less likely with environmental chemicals, as human exposure levels typically are much lower $[10,11]$. Thus, it seems reasonable to anticipate additive effects caused by real-life exposures to environmental chemicals in most cases.

Over the last decade, public authorities such as the US-Environmental Protection Agency (US-EPA), the Agency for Toxic Substances and Disease Registry (ATSDR), the World Health Organization (WHO), the non-food Committees of the European Commission, and the European Food Safety Authority (EFSA) have all made considerable progress towards developing pragmatic frameworks that are "fit for purpose" and tiered, to deal with combined exposure to multiple chemicals for risk assessment purposes [12-16].

The assumption that chemicals act additively and behave as if they were a simple dilution of each other has resulted in the development of methods for cumulative risk assessment using various approaches: Hazard Index (HI), point of departure index (PODI), relative potency factors (RPF) and toxicity equivalency factors (TEF). These relatively simple tools form a good basis for development of the future paradigm for MRA. 
67 Currently, EFSA is compiling the available knowledge on methodologies used to perform mixture risk 68 assessments for human health, animal health and ecological mixture risk assessment [17]. They present a tiered approach to a component based mixture risk assessment using principles of dose addition. HI calculation is considered a first tier summing hazard quotients (HQ) for all components regardless of toxicological endpoint. In the next tier, a refined HI approach takes into consideration that not all the components have the same adverse effect/target organ. A target organ toxicity dose is derived for each endpoint to estimate an end-point-specific Hazard Index [17].

However, as we see data gaps on single chemicals as the major bottleneck for MRA, we suggest a more specific workflow that allows the use of predicted or estimated hazard and exposure data for data-poor chemicals. The philosophy behind this is that risk assessment of chemicals would be greatly improved by taking mixture effects into account, even if great uncertainties are introduced for data-poor chemicals, and that this is better than doing nothing.

\subsection{Probabilistic models for exposure and hazard}

Real-life human exposure is highly variable and temporally dynamic, both at the individual and population levels. In spite of this, human exposures via the food is traditionally estimated using average intake levels of the chemicals, which is a deterministic method that does not incorporate information about the variability of real exposures. In order to reflect a more realistic exposure scenario, probabilistic exposure models are currently being developed and used for MRA $[3,18,19]$. In the same way, new tools for probabilistic hazard assessment are also being developed, which can be very useful for data-rich chemicals such as pesticides. However, for the vast majority of environmental or industrial chemicals, there is a lack of toxicity and exposure data; and since it is unrealistic to obtain 'perfect data' for most chemicals within the foreseeable future, probabilistic models may not be the best way forward for a pragmatic MRA of real-world mixtures 
within foreseeable future. This means that we currently are forced to perform MRA based on 'average' exposure and hazard data.

\subsection{Bottlenecks for mixture risk assessment}

One major challenge for MRA is that exposure to multiple chemicals currently is considered under different pieces of legislation, also known as regulatory silos, such as REACH or the EU pesticides Regulation [20,21]. Typically, humans are exposed to multiple chemicals from multiple sources, which can give rise to mixture effects, i.e. they can elicit similar effects or exhibit the same mode of action [22]. However, these chemicals may be risk assessed, risk managed or regulated by different authorities based on the legislations to which the chemicals belong. Thus, legal mandates for performing MRA across regulatory silos and legislations are needed, as are pragmatic MRAs allowing for cross-silos assessments.

Data gaps for individual chemicals seem to be a major issue when it comes to dealing with MRA, both regarding hazard and exposure data. In an attempt to address the lack of toxicity data, we suggest a pragmatic approach with a focus on how to derive information on hazards in cases where data are scarce and on methodologies for grouping of chemicals for MRA.

\section{A pragmatic approach for mixture risk assessment}

We have constructed a decision tree outlining a step-by-step procedure for MRA based on the use of Hazard Index (HI) (Fig. 1). This approach can be used to obtain a conservative (cautious) starting level for overall conclusions, and only progress to more refined methods including effect-based grouping of chemicals in cases where an initial concern for human toxicity is identified. This approach differs from previously suggested 'tiered' approaches [14,23], in which crude estimates with a high degree of caution are performed at early tiers in data poor situations, whereas higher tiers use more refined approaches with increasing data demand and higher certainty. However, since mixtures will usually contain both data-rich and data-poor 
chemicals, we need to be able to derive a HI for mixtures of chemicals with information of various refined

118 levels.

119 Our suggestion for a pragmatic MRA approach is shown in Figure 1 and described below. The numbers refer to the steps outlined in the figure.

Before starting the MRA, the challenge of the specific mixture is investigated and relevant questions relating to for instance the exact composition of the mixture and the potential for concomitant human exposure is addressed.

1. First, an evaluation of data availability is made for individual chemicals from the mixture and, if possible, for the whole mixture, i.e., is relevant hazard information on the individual chemicals available (1A? If possible, NOAELs or similar hazard measures from in vivo experiments are used to set a reference value (RfV) for each chemical. If not possible, toxicity may be estimated by using relevant in vitro information in combination with a human exposure level (see example in [24, this issue]). If no experimental data exists, read-across data or quantitative QSAR data may be applied and toxicity values for similar chemicals can be used as a surrogate. Next, do human exposure data exist (1B)? Do reliable human biomonitoring data exist? Have the sources been identified? Can exposure via the food be estimated from contaminant data for specific food items? Furthermore, it needs to be considered whether mean or median exposure values, or 95\% percentiles should be applied in the given situation.

2. Hazard quotients (HQs) are calculated as the ratio between exposure estimate and RfV for a given substance based on the collected data in step 1. We suggest allowing the use of HQs calculated from relevant in vitro data and for instance human biomonitoring data. An example of this can be found in Johansson et al. (2019) in this issue. If the HQ is above or close to 1 for any single chemical, the decision to regulate is handed over to the regulatory bodies. If all HQs are less than 1, proceed to step 3.

3. At this step, it is considered whether deviations from dose additivity can be expected. Are there reasons to suspect that interaction between the chemicals will occur by toxicodynamic or toxicokinetic interactions? 
144 Are CYP450 interactions likely at the present exposure levels e.g. due to CYP450 induction? If no deviations

145 from dose-addition are expected, proceed to step 4. However, if deviations can be expected, a case-by-case 146 MRA should be applied.

148 4. Assuming dose additivity, a calculation of a rough and non-refined HI is performed. At this step, all 149 chemicals are added in the same formula, irrespective of the type of toxicity they are causing. The rationale 150 for summing up all hazards irrespective of effects is based on considering the human body as one complex 151 organism, as well as the hypothesis that various toxicities are often interrelated. A major limitation of this 152 step is a high degree of inherent uncertainties when setting RfV and exposure estimates for each chemical in 153 the mixture. In addition, it is acknowledged that use of the dose-addition principle is not precise for chemical 154 mixtures for which different endpoints have been used for deriving RfVs. If the calculated HI exceeds 1, it 155 will be necessary to group the chemicals according to their specific toxicities (step 5). In cases where the HI 156 becomes less than 1 , proceed to step 6.

157

158 5. For mixtures with a non-refined $\mathrm{HI}$ higher than 1 , it is necessary to group the chemicals according to effect 159 or mode-/mechanism-of-action using available in vivo, in vitro or in silico data (see below). For each group 160 of chemicals, a more specific and refined $\mathrm{HI}$ is calculated. If any of these HI's exceeds 1, regulatory action 161 should be decided upon by the authorities. For those HI’s that are less than 1, proceed to step 6.

163 6. For HIs less than 1, with or without grouping, it should be considered whether other sources of the same 164 chemicals exist or if other chemicals are present that are likely to contribute to the mixture effect. If so, it 165 should be accounted for as described in step 7. If not, no human risk is anticipated for the mixture. 
7. If other chemicals or sources are expected to contribute to the mixture effect, the HI cut-off of 1 should be adjusted to take this extra contribution (X) into account, i.e. to a cut-off of (1-X). For example, for a specific mixture obtained via the diet, it may be estimated that humans are exposed to the same chemicals from other sources such as via inhalation or via dermal absorption and that these would contribute with $30 \%$ of the acceptable risk. Then, the cut-off for acceptable risk for the evaluated mixture is adjusted to $70 \%$ (0.7) instead of 1 . If the $\mathrm{HI}<(1-\mathrm{X})$ where $\mathrm{X}$ designates the extra contribution, then no human risk is anticipated. In this example, a HI below 70\% would be deemed acceptable. If the calculated $\mathrm{HI}$ exceeds (1-X), it will be necessary to group the chemicals according to their specific toxicities as described in step 5 . Thus, using this example a HI above 70\% would lead to (re-) evaluation of the possibility of grouping mixture components according to shared effects or modes of action (step 5). If any of the HI's exceeds the adjusted cut-off value after grouping of the chemicals, regulatory action should be decided upon by the authorities. If all HI's are still below the adjusted cut-off value after grouping, then no human risk is anticipated for the mixture.

\section{Grouping of chemicals}

An essential step in MRA is the grouping of chemicals according to their effects or mode/mechanism of action (step 5) using relevant animal and non-animal data. A standardized methodology for grouping of chemicals based on target organ toxicities has been proposed by Adams et al. [25]. This corresponds to the second tier proposed for component based mixture risk assessment in EFSAs draft guidance document [17]. Furthermore, EFSA has presented cumulative assessment groups (CAGs) for pesticides with effects on either the nervous system or the thyroid hormone system [26], and CAGs for several other target organs have been proposed by Nielsen et al. 2012 [27]. This approach is limited to chemicals for which in vivo data exist. As an alternative, grouping based on common modes of action may include grouping ofcompounds that act on the same adverse outcome pathways (AOPs). An AOP describes a sequence of events from initial interaction(s) of a stressor with a biomolecule (molecular initiating event), causing perturbations which can progress through a series of intermediate key events and culminate in an adverse outcome [28]. In the future, complex AOP networks may be a preferred methodology for grouping of chemicals, but requires the 
development of a more densely populated AOP wiki (https://aopwiki.org/). Until such time, we suggest to use empirical in vivo and in vitro data, read-across as well as computational methods such as QSAR models or integrative systems biology. These latter non-test methods can be used to predict inherent hazards or modes/mechanisms of action and to group the chemicals in cases where no experimental data exist.

\subsection{Grouping according to experimental data}

Toxicological signatures (or profiles) of chemicals obtained from in vitro studies can be used for grouping. Profiling of chemicals across a panel of in vitro tests was previously applied to explore whether structurally related chemicals share the same mechanism of action. As shown in Fig. 2A, a range of bisphenols had a qualitative similar profile across a panel of assays for endocrine activity [29] and should therefore be grouped together, whereas a number of fluorinated chemicals should be divided into different subgroups based on their specific in vitro signatures [30].

Toxicological signatures based on omics data such as metabolomics, transcriptomics or proteomics can be established from either in vivo or in vitro studies. By using omics data, chemicals with specific modes/mechanisms of action can be identified based on the signature of affected metabolites, genes or proteins $[31,32]$ and this can be used to decide on grouping of chemicals or not.

\subsection{Grouping based on computational tools}

Another grouping approach is the use of computer models known as QSARs (Quantitative Structure-Activity Relationships). QSARs estimate the relationship between the structure of chemical substances and another property such as the ability to cause toxic effects. QSAR models can therefore be used to predict hazards of chemicals with no or insufficient test data. Figure 2B depicts an example of a QSAR screening performed on 2,076 chemicals from food contact materials, including predictions for genotoxic carcinogenicity, mutagenicity, and developmental toxicity [33]. Detailed information on the applied decision-algorithms and the performance of the individual models has been described previously [34]. The figure shows the number of chemicals predicted to be active for each hazard as well as the overlap between the three hazards, which 
provides a basis for grouping of the chemicals. The free Danish QSAR database can be searched for 220 estimates related to e.g. metabolism and toxicity for more than 600,000 chemicals (http://qsar.food.dtu.dk/).

221 This database is part of the OECD QSAR Toolbox, a software application which incorporates information 222 and tools from various sources (https://www.qsartoolbox.org/home).

223 'Read across' is one of the most commonly used alternative approaches for filling data gaps in registrations 224 for REACH and entails the use of relevant information from analogous chemicals to predict for instance 225 hazards for the chemical in question [35,36]. Thus, chemicals whose toxicological properties are likely to be 226 similar or follow a regular pattern as a result of structural similarity may be considered a category [36]). The 227 read-across hypothesis needs to be justified by scientifically credible explanations and sufficient supporting information such as information from QSARs or experimental data addressing specific aspects of the readacross hypothesis [36]. Many tools for read-across assessment are available, and in this regard the OECD Toolbox is most advanced in terms of addressing current regulatory needs [37].

\subsection{Grouping based on a systems biology approach}

A third approach for grouping chemicals is by use of systems biology approaches (Fig 2C). Advanced bioinformatics tools can be used to gain mechanistic knowledge by taking advantage of already existing information about associations between human targets and chemicals. For example, Kongsbak et al [38,39], retrieved information for known chemical-protein associations from two publicly available databases, the

237 Comparative Toxicogenomics Database (CTD) [40] and ChemProt 2.0 [41]. This type of analysis gives an 238 overview of known and potential human targets for the chemicals of interest. If chemicals in a mixture then 239 turn out to target the same molecules or are significantly associated with the same disease, or affect the same 240 signaling pathway, it is likely that these chemicals can be allocated to the same group for MRA. In Figure 241 2C, it is illustrated how mancozeb can be excluded from the group, as this chemical belongs to its own 242 network.

243 In conclusion, there are several ways of grouping chemicals, which should be used and explored in more 244 detail for MRA of chemicals. 


\section{Perspectives}

Although several proposals have been published for how to handle risk assessments of chemical mixtures, no consensus has been reached. Given the complexity of MRA and the potential human health risk not accounted for, we propose a pragmatic approach. On the one hand this approach will improve on chemical risk assessment, but on the other require that we accept some uncertainties in the evaluations. The HI is not necessarily an accurate indicator of risk as it is based on hazard and exposure values that are subject to uncertainty. Nevertheless, it provides a low-input, straightforward risk assessment tool that is an improvement compared to the alternative of performing single-chemical risk assessments.

An important limitation of current MRA is the lack of data on individual chemicals, both with regard to human exposure and toxicity data. To date, only a minor fraction of the many thousands of chemicals in current use have been evaluated and allocated an ADI, TDI, NOAEL, or similar. There is thus an urgent need to address this lack of toxicity data and a way forward could be to develop alternative ways of risk assessing chemicals based on for instance defined panels of in vitro models, or computational tools such as QSARs and physiologically-based kinetic modelling.

Our proposal is designed to prevent bottlenecks such as lack of data on individual chemicals or complex reallife exposures stopping us from performing a pragmatic MRA of chemicals.

\section{Conflicts of interest}

265 All authors declare no conflicts of interest.

\section{References}

267 Papers of particular interest have been highlighted as:

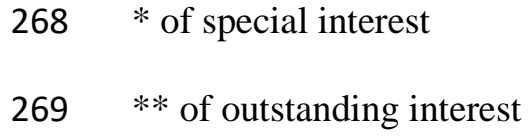


* [1] Svingen, T., Vinggaard, A.M. 2016. The risk of chemical cocktail effects and how to deal with the issue. Journal of Epidemiology and Community Health. Editorial, 70 (4), 322-323.

[2] Bernhardt, E.S., Rosi, E.J., Gessner, M.O. 2017. Synthetic chemicals as agents of global change. Front Ecol Environ 15(2), 84-90, doi:10.1002/fee.1450

[3] EFSA, 2013. International Frameworks Dealing with Human Risk Assessment of Combined Exposure to Multiple Chemicals, EFSA Journal. Wiley-Blackwell. https://doi.org/10.2903/j.efsa.2013.3313

* [4] Kortenkamp, A., Backhaus, T., Faust, M., 2009. European Commission. State of the Art Report on Mixture Toxicity. http://ec.europa.eu/environment/chemicals/effects/pdf/report_mixture_toxicity.pdf (accessed April 2018).

[5] Silva, E., Rajapakse, N., Kortenkamp, A., 2002. Something from “nothing”--eight weak estrogenic chemicals combined at concentrations below NOECs produce significant mixture effects. Environ. Sci. Technol. 36, 1751-1756.

[6] Conley, J.M., Lambright, C.S., Evans, N., Cardon, M., Furr, J., Wilson, V.S., Gray Leon Earl, J., 2018. Mixed “Antiandrogenic” Chemicals at Low Individual Doses Produce Reproductive Tract Malformations in the Male Rat. Toxicol. Sci. kfy069-kfy069.

[7] Hass, U., Scholze, M., Christiansen, S., Dalgaard, M., Vinggaard, A.M., Axelstad, M., Metzdorff, S.B., Kortenkamp, A., 2007. Combined exposure to anti-androgens exacerbates disruption of sexual differentiation in the rat. Environ. Health Perspect. 115 Suppl 1, 122-128. https://doi.org/10.1289/ehp.9360

[8] Howdeshell, K.L., Hotchkiss, A.K., Gray Jr, L.E., 2017. Cumulative effects of antiandrogenic chemical mixtures and their relevance to human health risk assessment. Int. J. Hyg. Environ. Health 220, 179188. https://doi.org/S1438-4639(16)30217-6 [pii]

[9] Tannenbaum, C., Sheehan, N.L., 2014. Understanding and preventing drug-drug and drug-gene interactions. Expert Rev. Clin. Pharmacol. 7, 533-544. https://doi.org/10.1586/17512433.2014.910111

[10] Rappaport, S.M., Barupal, D.K., Wishart, D., Vineis, P., Scalbert, A., 2014. The blood exposome and its role in discovering causes of disease. Environ. Health Perspect. 122, 769-774. https://doi.org/10.1289/ehp.1308015 
* [11] Boobis, A., Budinsky, R., Collie, S., Crofton, K., Embry, M., Felter, S., Hertzberg, R., Kopp, D., Mihlan, G., Mumtaz, M., Price, P., Solomon, K., Teuschler, L., Yang, R., Zaleski, R., 2011. Critical analysis of literature on low-dose synergy for use in screening chemical mixtures for risk assessment. Crit. Rev. Toxicol. 41, 369-383. https://doi.org/10.3109/10408444.2010.543655

* [12]ATSDR, 2018. Framework for Assessing Health Impacts of Multiple Chemicals and Other Stressors. Atlanta, GA. https://www.atsdr.cdc.gov/interactionprofiles/ipga.html

[13] EFSA, 2008. Scientific Opinion of the Panel on Plant Protection Products and their Residues (PPR Panel) on a request from the EFSA evaluate the suitability of existing methodologies and, if appropriate, the identification of new approaches to assess cumulative and sy. EFSA J. 1-85. https://doi.org/10.2903/j.efsa.2008.705

[14] Meek, M.E., Boobis, A.R., Crofton, K.M., Heinemeyer, G., Raaij, M. V, Vickers, C., 2011. Risk assessment of combined exposure to multiple chemicals: A WHO/IPCS framework. Regul. Toxicol. Pharmacol. https://doi.org/S0273-2300(11)00063-8 [pii]

[15] SCHER, SCENIHR, SCCS, 2012. Opinion on the Toxicity and Assessment of Chemical Mixtures 1-50. https://doi.org/10.2772/37863

[16] US-EPA, 2008. Concepts, Methods, and Data Sources for Cumulative Health Risk Assessment of Multiple Chemicals, Exposures and Effects: A Resource Document.

[17] EFSA Scientific Committee, 2018. Draft guidance on harmonised methodologies for human health, animal health and ecological risk assessment of combined exposure to multiple chemicals. Draft in public consultation, June 2018.

[18] Kienzler, A., Berggren, E., Bessems, J., Bopp, S., Van Der Linden, S., Worth, A., 2017. Assessment of Mixtures - Review of Regulatory Requirements and Guidance. https://doi.org/10.2788/84264

[19] Euromix project. http://www.euromixproject.eu/

[20] Evans, R.M., Martin, O. V, Faust, M., Kortenkamp, A., 2016. Should the scope of human mixture risk assessment span legislative/regulatory silos for chemicals? Sci. Total Environ. 543, 757-764. https://doi.org/10.1016/j.scitotenv.2015.10.162 [doi]

[21] Kienzler, A., Bopp, S.K., van der Linden, S., Berggren, E., Worth, A., 2016. Regulatory assessment of 
chemical mixtures: Requirements, current approaches and future perspectives. Regul. Toxicol. Pharmacol. 80, 321-334. https://doi.org/10.1016/j.yrtph.2016.05.020

[22] Hadrup, N., Pedersen, M., Skov K., Hansen, N.L., Berthelsen, L.O., Kongsbak, K., Boberg, J., Dybdahl, M., Hass, U., Frandsen, H., Vinggaard, A.M. 2016. Perfluorononanoic acid in combination with 14 chemicals exerts low dose mixture effects in rats. Arch. Toxicol., 90 (3), 661-675.

[23] Price, P., Han, X., Junghans, M., Kunz, P., Watts, C., Leverett, D., 2012. An application of a decision tree for assessing effects from exposures to multiple substances to the assessment of human and ecological effects from combined exposures to chemicals observed in surface waters and waste water effluents. Environ. Sci. Eur. 24, 34. https://doi.org/10.1186/2190-4715-24-34

[24] Johansson, H.K.L., Boberg, J., Dybdahl, M., Axelstad, M., Vinggaard, A.M. Chemical risk assessment based on in vitro and human biomonitoring data: A case study on thyroid toxicants. Current Opinion of Toxicology, This issue.

[25] Adams, V.H., McAtee, M.J., Johnson M.S., 2017. Implementation of the Basic Hazard Index Screening for Health Risks Associated with Simultaneous Exposure to Multiple Chemicals Using a Standardized Target Organ and Systems Framework. Integrated Environmental Assessment and Management 13(5), 852-860

[26] EFSA 2013. Scientific Opinion on the identification of pesticides to be included in cumulative assessment groups on the basis of their toxicological profile. EFSA PPR panel. EFSA Journal 11(7):3293.

[27] Nielsen, E., Nørhede, P., Boberg, J. et al. 2012. Identification of cumulative assessment groups of pesticides. External scientific report submitted to EFSA. http://www.efsa.europa.eu/en/supporting/pub/269e.htm

[28] OECD 2016. OECD Environment, Health and Safety Publications. Series on Testing and Assessment No. 233. USERS’ HANDBOOK SUPPLEMENT TO THE GUIDANCE DOCUMENT FOR DEVELOPING AND ASSESSING AOPs. ENV/JM/MONO(2016)12.

[29] Rosenmai, A.K., Dybdahl, M., Pedersen, M., van Vugt-Lussenburg, B.M.A., Wedebye, E.B., Taxvig, C., Vinggaard, A.M., 2014. Are structural analogues to bisphenol a safe alternatives? Toxicol. Sci. 139. 
https://doi.org/10.1093/toxsci/kfu030

[30] Rosenmai, A.K., Taxvig, C., Svingen, T., Trier, X., van Vugt-Lussenburg, B.M., Pedersen, M., Lesne, L., Jegou, B., Vinggaard, A.M., 2016. Fluorinated alkyl substances and technical mixtures used in food paper-packaging exhibit endocrine-related activity in vitro. Andrology 4, 662-672. https://doi.org/10.1111/andr.12190 [doi]

[31] van Ravenzwaay, B., Galay Burgos, M., Vrijhof, H., 2012. Use of 'omics to elucidate mechanism of action and integration of 'omics in a systems biology concept. Mutat. Res. Toxicol. Environ. Mutagen. 746, 95-96. https://doi.org/10.1016/J.MRGENTOX.2012.04.004

[32] Brockmeier, E.K., Hodges, G., Hutchinson, T.H., Butler, E., Hecker, M., Tollefsen, K.E., GarciaReyero, N., Kille, P., Becker, D., Chipman, K., Colbourne, J., Collette, T.W., Cossins, A., Cronin, M., Graystock, P., Gutsell, S., Knapen, D., Katsiadaki, I., Lange, A., Marshall, S., Owen, S.F., Perkins, E.J., Plaistow, S., Schroeder, A., Taylor, D., Viant, M., Ankley, G., Falciani, F., 2017. The Role of Omics in the Application of Adverse Outcome Pathways for Chemical Risk Assessment. Toxicological Sciences, 158(2), 2017, 252-262.

[33] Rosenmai, A.K., Bengtström, L., Taxvig, C., Trier, X., Petersen, J.H., Svingen, T., Binderup, M.-L., Barbara Medea Alice, V.V.-L., Dybdahl, M., Granby, K., Vinggaard, A.M., 2017. An effect-directed strategy for characterizing emerging chemicals in food contact materials made from paper and board. Food Chem. Toxicol. 106. https://doi.org/10.1016/j.fct.2017.05.061

[34] Wedebye, E.B., Dybdahl, M., Nikolov, N.G., Jónsdóttir, S.T., Niemelä, J.R., 2015. QSAR screening of 70,983 REACH substances for genotoxic carcinogenicity, mutagenicity and developmental toxicity in the ChemScreen project. Reprod. Toxicol. 55. https://doi.org/10.1016/j.reprotox.2015.03.002

[35] Ball, N., Cronin, M.T., et al, 2016. Toward Good Read-Across Practice (GRAP) guidance. ALTEX 33(2):149-66. doi: 10.14573/altex.1601251.

[36] ECHA, 2017. Read-across assessment framework (RAAF).

[37] Patlewicz, G., Helman, G., Pradeep, P., Shah, I., 2017. Navigating through the minefield of read-across tools: A review of in silico tools for grouping. Computational Toxicology 3, 1-18.

[38] Kongsbak, K., Hadrup, N., Audouze, K., Vinggaard, A.M., 2014a. Applicability of computational 
systems biology in toxicology. Basic Clin. Pharmacol. Toxicol. 115, 45-49. https://doi.org/10.1111/bcpt.12216 [doi]

[39] Kongsbak, K., Vinggaard, A.M., Hadrup, N., Audouze, K., 2014b. A computational approach to mechanistic and predictive toxicology of pesticides. ALTEX 31, 11-22. https://doi.org/10.14573/altex.1304241 [doi]

[40] Davis, A.P., Murphy, C.G., Johnson, R., Lay, J.M., Lennon-Hopkins, K., Saraceni-Richards, C., Sciaky, D., King, B.L., Rosenstein, M.C., Wiegers, T.C., Mattingly, C.J., 2013. The Comparative Toxicogenomics Database: update 2013. Nucleic Acids Res. 41, D1104-14. https://doi.org/10.1093/nar/gks994 [doi]

[41] Kim Kjaerulff, S., Wich, L., Kringelum, J., Jacobsen, U.P., Kouskoumvekaki, I., Audouze, K., Lund, O., Brunak, S., Oprea, T.I., Taboureau, O., 2013. ChemProt-2.0: visual navigation in a disease chemical biology database. Nucleic Acids Res. 41, D464-9. https://doi.org/10.1093/nar/gks1166 [doi]

\section{Figure legends}

Figure 1 . The proposed decision tree based on the use of the hazard index. The step-by-step procedure is described in detail in the text.

Figure 2. Examples of approaches to group chemicals. A) Profiling of chemicals using defined panels of in vitro tests. As shown, a range of bisphenols had a qualitative similar profile in a panel of assays for endocrine activity, while fluorinated chemicals could be divided into different groups based on the in vitro signature $[27,28]$. B) Venn diagram showing the number of chemicals predicted by QSAR to be active for each hazard as well as the overlap between the three hazards. C) Chemical-protein association network of five chemicals and their associated proteins. The colors of the proteins/genes indicate the primary function of the encoded protein. As seen from the figure, mancozeb does not share any associations with the four other chemicals, which means that this compound can be excluded from the mixture in a grouping exercise [34]. 